\title{
Memory conformity and the perceived accuracy of self versus other
}

\author{
Kevin Allan • J. Palli Midjord • Doug Martin • \\ Fiona Gabbert
}

Published online: 15 September 2011

(C) Psychonomic Society, Inc. 2011

\begin{abstract}
Here, we demonstrate that the decision to conform to another person's memory involves a strategic trade-off that balances the accuracy of one's own memory against that of another person. We showed participants three household scenes, one for $30 \mathrm{~s}$, one for $60 \mathrm{~s}$, and one for $120 \mathrm{~s}$. Half were told that they would encode each scene for half as long as their virtual partner, and half were told that they would encode each scene for twice as long as their virtual partner. On a subsequent two-alternative-forced choice (2AFC) memory test, the simulated answer of the partner (accurate, errant, or no response) was shown before participants responded. Conformity to the partner's responses was significantly enhanced for the 30-s versus the 60- and 120 -s scenes. This pattern, however, was present only in the group who believed that they had encoded each scene for half as long as their partner, even though the short-duration scene had the lowest baseline 2AFC accuracy in both groups and was also subjectively rated as the least memorable by both groups. Our reliance on other people's memory is therefore dynamically and strategically adjusted according to knowledge of the conditions under which we and other people have acquired different memories.
\end{abstract}

Keywords Memory conformity $\cdot$ Metacognition $\cdot$ Social cognition $\cdot$ Mental simulation

K. Allan $(\bowtie) \cdot J$. P. Midjord $\cdot$ D. Martin

School of Psychology, College of Life Sciences and Medicine, University of Aberdeen,

Aberdeen AB24 2UB, UK

e-mail: k.allan@abdn.ac.uk

F. Gabbert

Division of Psychology, School of Social and Health Sciences, University of Abertay,

Dundee, Scotland

\section{Introduction}

We often discuss and compare our memories of past events with one another, and during these social interactions, we may encounter accurate or errant information about the past. If this information is accepted, the accuracy of our own memory may be correspondingly improved or impaired, with consequences ranging from the trivial to the highly significant (confusion resulting from discussion between witnesses following the Oklahoma City bombing incident in April, 1995, is an often-cited example). Although conformity to another person's memory is forensically undesirable, it may nonetheless be a rational and, perhaps, even adaptive way for us to function socially in situations where we have a relative distrust of our own memory. Indeed, the ability to incorporate another person's perspective within one's own is arguably an essential feature of all social cognition (Macrae \& Bodenhausen, 2000). But how do we do this? In particular, how do we trade off the need to maintain a stable and reasonably accurate memory of our own while remaining open to another person's perspective on the past?

Prior work has tended to focus on different aspects of the trade-off, either by manipulating the perceived credibility of another individual's memory, as compared with one's own (e.g., French, Garry, \& Mori, 2011; Gabbert, Memon, \& Wright, 2007), or by manipulating how well one's own memory is actually operating (e.g., Baron, Vandello, \& Brunsman, 1996; Roediger, Meade, \& Bergman, 2001). These two approaches have produced complementary findings indicating that we become more reliant on other people's memory when they are perceived as more credible than we are, and also when our own memory begins to function less accurately. Each of these approaches, however, illustrates a quite special case and not a more general principle of the 
regulatory process at work when we share memories of the past with one another. That is, under some circumstances, we may indeed conform more to highly credible sources, but surely not without respect to how well our own memory is functioning. And under some circumstances, we may indeed conform more when our memory begins to fail us, but surely not without regard to the credibility of the other person. Existing approaches have therefore revealed special cases consistent with the general principle that beliefs about memory in one's self and other people can trade off dynamically. Here, for the first time to our knowledge, we directly demonstrate the full trade-off in operation. Before describing our approach, we briefly review past work leading to our present study.

Interest in memory conformity, also termed the social contagion of memory (e.g., Meade \& Roediger, 2002; Roediger et al., 2001), was reawakened in the mid-1990s, some 40 years after initial reports by Deutsch and Gerard (1955). Schneider and Watkins (1996) showed that when individuals swap information about a shared past experience, this can readily lead to conformity. We and others have since shown that information encountered during a social interaction produces stronger distorting effects upon memory than do comparable, nonsocial sources of information (e.g., Gabbert, Memon, Allan, \& Wright, 2004; Meade \& Roediger, 2002; but see Bodner, Musch, \& Azad, 2009), and recent work has focused on various factors that moderate these distortions. For example, individuals are particularly susceptible to memory conformity when collaborating with a partner who is perceived as relatively more "powerful" (Skagerberg \& Wright, 2009). Similar effects also emerge when the closeness of the relationship between individuals within a dyad systematically varies across conditions (French, Garry, \& Mori, 2008; Hope, Ost, Gabbert, Healey, \& Lenton, 2008).

Of most relevance to the present work, it has also been reported that the tendency to conform is enhanced if we believe another person's memory is likely to be better than our own. Gabbert et al. (2007) allowed all their participants to view images for the same fixed duration, but they told half their participants that they had encoded the images for longer than their partner and the remainder that they had encoded the images for less time than their partner. Participants who believed that they had encoded images for less time conformed more to their partner than did participants in the other group, even though participants' recall accuracy was equivalent in both groups. French et al. (2011) recently produced evidence of a similar pattern obtained by manipulating participants' beliefs about the relative visual acuity of their partner. Complementing this work in which the relative credibility of participants' partner was manipulated, in a parallel series of studies, it has been shown that increases and decreases in the accuracy of one's own memory can systematically reduce or elevate, respectively, the extent to which one is willing to conform to another person's judgments (e.g., Baron et al., 1996; Roediger et al., 2001; see also Tousignant, Hall, \& Loftus, 1986). These two complementary lines of work have informed Wright and colleagues' recent proposal (Wright, London, \& Waechter, 2010; Wright \& Schwartz, 2010) that conformity motivated by the desire to maintain an accurate memory - that is, deriving from an informational social influence (Cialdini \& Goldstein, 2004) - results from a combination of beliefs about our own memory and our partner's memory. Here, we provide a novel and direct test of this view by integrating the two strands of work on partner credibility and one's own accuracy within a single approach.

Our approach involved testing people's memories for busy scenes, using the virtual partner method of Allan and Gabbert (2008). This method allowed simulated responses from a virtual partner to be generated and shown to participants. Here, we manipulated the actual duration that participants were given to encode each scene (30,60, and 120 s), but we informed half of the participants that they would view each scene for half as long as their (virtual) partner, while telling the remainder that they would view each scene for twice as long as their partner. Hence, we could simultaneously manipulate our participants' encoding duration as well as their beliefs regarding their partner's encoding duration. When combined, these two manipulations allowed us to determine whether belief in the relative quality of someone else's memory always would affect conformity, in which case we should observe that participants in the half group conformed more often regardless of how long they had to encode, as compared with participants in the twice group. Alternatively, our reliance on other people might depend on how well our own memory is operating, in which case we might observe that, regardless of group, participants conformed more to information provided about images encoded for shorter versus longer durations. But if conformity truly results from a strategic combination of beliefs about self and other, as we argue here and as was suggested by Wright and colleagues (Wright et al., 2010; Wright \& Schwartz, 2010), we should instead observe that brief encoding durations enhanced conformity only in individuals who believed that they had encoded for half as long as their partner. Therefore, we specifically predicted an effect of encoding duration upon conformity that would differ according to (i.e., that would interact with) the half versus twice participant-group factor.

\section{Method}

Participants

In a large computer lab housing networked PCs, we ran 96 undergraduate psychology students in return for course 
credit. They attended in groups of between 6 and 12 individuals ( 38 of them male; mean age, 20.2 years, $S D=$ 2.1; half group, $n=48$; twice group, $n=48$ ).

\section{Stimuli}

Each participant viewed three color pictures of household scenes (Allan \& Gabbert, 2008; Meade \& Roediger, 2002). The encoding durations used for all participants were identical. One picture was viewed for $30 \mathrm{~s}$, one for $60 \mathrm{~s}$, and one for $120 \mathrm{~s}$. The mapping of picture to encoding duration was counterbalanced across participants, and the order in which the duration manipulation was given was also newly randomized for each participant. Following picture encoding, we asked our participants to perform a distractor task where they had to rate their spontaneous use of visual imagery (SUIS) in different real-life contexts (Reisberg, Pearson, \& Kosslyn, 2003). This was done purely to mask the fact that encoding durations did not actually differ in the half versus twice groups, and we do not report any analyses based on these data.

The memory test involved a two-alternative forced choice (2AFC) procedure (see Allan \& Gabbert, 2008) where 30 specific questions were asked for each scene (e.g. "What color was the bathroom carpet? Red or blue?"). The order of the resulting 90 questions was newly randomized for each participant. Participants were told that they should respond after their partner. Each trial began with a fixation cross, replaced after $1 \mathrm{~s}$ by the $2 \mathrm{AFC}$ question and answers and then a fixed 3-s gap in which the partners' response was notionally made. Before providing their own answer the participants were shown onscreen the answer chosen by their partner, as indicated by an underline underneath one of the $2 \mathrm{AFC}$ answers. On one third (i.e., 30 of 90) of the trials, the partner's answer was accurate; on one third, it was errant; and on one third, no response was shown. At random, we split the set of 30 questions for each picture into three sets of 10 , and across participants, we ensured that each subset of 10 questions received each kind of postevent information (PEI) - that is, an accurate response, errant response, or no response, equally often.

\section{Procedure}

Participants were randomly allocated to the between subjects' half- and twice-as-long partner-encoding conditions, and we ensured that participants in the different conditions were segregated within the lab. Participants were first told that they would perform a collaborative memory task along with one of the other participants, who would be picked at random by the computer and who would remain their partner throughout the whole experiment. They were then informed that the experiment examined the relationship between individual differences in mental imagery ability and collaborative behavior. They were then instructed that they would view three scenes for varying lengths of time but that they would view each scene either for half as long or twice as long as their partner. They were further informed that however long they viewed a particular scene for, their partner would have viewed it for either half as long or twice as long. As was noted above, the actual viewing durations for all participants were identical and were set at 30,60, and $120 \mathrm{~s}$. Participants were told that they would not know, in advance of each scene, how long the viewing time would be and that they should try to maintain attention to each scene for as long as it remained onscreen. After viewing all three scenes, onscreen instructions regarding the SUIS task were given, and participants were told to wait, after they had completed the SUIS, until further instructions were given. The average study-test interval (i.e., the average time to complete the imagery tasks) was $\sim 5 \mathrm{~min}$. Immediately prior to the memory test, the participants were reminded by an onscreen message about whether they had encoded each scene for half as long or twice as long as their partner. They were also informed that their goal during the memory test was to provide accurate responses and that we would not be assessing the collaborative accuracy of the dyad in which they were participating. This was made explicit to the participants in order to emphasize informational motivations to conform and to minimize any strategy that would emphasize response conformity (Bodner et al., 2009). They were then informed by onscreen instructions that they were required to respond after their partner on each trial and that the partner's answer would be underlined. We also told the participants that on some trials, a partner might not respond quickly enough and so it was possible that no response option would be underlined, in which case the participants should give their own answer. The participant was to use either the "Q" or "P" key to indicate whether they remembered the detail shown as the left ("Q") or right ("P") $2 \mathrm{AFC}$ response option. In total, the full experiment took $\sim 45 \mathrm{~min}$ to complete.

As a final manipulation check, to verify that the different encoding durations systematically altered participants beliefs about the functioning of memory, we asked participants, after completing the memory test, to indicate which image they had the best memory for and which image they had the worst memory for.

\section{Results}

Manipulation check

First. we examined the manipulation check data to determine whether memory for images encoded for the long (120-s) duration was consistently rated as best and whether image memory for the short duration (30 s) was 
consistently rated as worst. Table 1 shows the percentage of participants in each group who rated their memory for the short-, medium-, and long-duration images as best or worst. In both groups, there was a significant association between perceived memory quality (i.e., best vs. worst memory) and encoding duration [half group, $\chi^{2}(2)=33.62, p<.001$; twice group, $\left.\chi^{2}(2) 26.16, p<.001\right]$.

\section{Baseline accuracy}

The participants' ratings of best/worst memory were consistent with the baseline accuracy of $2 \mathrm{AFC}$ recognition when the virtual partner gave no response (see Fig. 1). A mixed-design repeated measures ANOVA (group [half/ twice]; encoding duration [30 s/60 s/120 s]) was used to examine the baseline performance. Note that the dependent variable (DV) here, and in each of the subsequent analyses reported below, is correct $2 \mathrm{AFC}$ performance. We observed a significant main effect of encoding duration, $F(2,188)=$ 20.17, $p<.001, \eta_{\mathrm{p}}{ }^{2}=.177, M S E=177.1$, but this did not interact with the group factor, $F=0.64$. Within each group, baseline was highest for the long duration, lowest for the short duration, and in-between for the medium duration, minimum $t(95)=2.80, p=.006, S E M=2.0$. These analyses confirm that as encoding duration increases, so does $2 \mathrm{AFC}$ accuracy in the baseline (no PEI) condition and that an identical pattern is present for both groups. The effect of encoding duration upon the baseline data therefore mirrors the effect of encoding duration upon the participants' subjective ratings of their memory quality.

\section{Memory conformity}

The conformity data are shown separately for each group in Figs. 2 and 3, which depict performance following exposure to accurate and errant PEI after zeroing by subtracting performance in the corresponding baseline noPEI conditions from Fig. 1. A comparison of Figs. 2 and 3 reveals a consistent effect of encoding duration within the half group. As encoding duration decreases, divergence from baseline increases, and moreover, a similar pattern appears to be present in the accurate and the errant PEI conditions. This pattern suggests that reduced encoding duration is enhancing reliance upon the memory of one's partner specifically in the half group. To determine whether encoding duration does, in fact, exert a differential effect upon conformity in each participant-group, we initially employed a mixed-design ANOVA that compared the effect of encoding duration $(30 \mathrm{~s} / 60 \mathrm{~s} / 120 \mathrm{~s})$ upon the DV of correct 2AFC performance in all three PEI conditions (accurate/errant/baseline) as a function of group (half/ twice). This ANOVA produced main effects of encoding duration, $F(2,188)=21.64, p<.001, \eta_{\mathrm{p}}{ }^{2}=.187, M S E=$ 279.6, and of the PEI factor, $F(2,188)=94.37, p<.001$, $\eta_{\mathrm{p}}{ }^{2}=.501, M S E=327.1$, as well as a two-way interaction between encoding duration and PEI, $F(4,376)=3.17, p=$ $.014, \eta_{\mathrm{p}}{ }^{2}=.033, M S E=150.0$. All of these effects were, however, qualified by the significant three-way interaction between encoding duration, PEI, and group, $F(4,376)=$ $3.83, p=.005, \eta_{\mathrm{p}}{ }^{2}=.039, M S E=150.0$, that we specifically predicted.

To further elucidate this three-way interaction, we first determined whether group differences in the effect of encoding duration upon correct $2 \mathrm{AFC}$ performance were specific to the accurate or to the errant PEI conditions. For accurate PEI versus baseline, the subsidiary ANOVA (group [half/twice]; encoding duration [30 s/60 s/120 s]; PEI [accurate/baseline]) revealed main effects of encoding duration, $F(2,188)=14.4$, $p<.001, \eta_{\mathrm{p}}^{2}=.133, M S E=232.6$, and PEI, $F(1,94)=$ $47.45, \eta_{\mathrm{p}}{ }^{2}=.335, M S E=177.1$, as well as an encoding duration $\times$ PEI interaction, $F(2,188)=6.36, p=.002, \eta_{\mathrm{p}}{ }^{2}=$ $.063, M S E=122.0$, but we did not observe any significant effects involving the group factor (maximum $F=2.07, p=$ .12 , for the encoding duration $\times$ PEI $\times$ group interaction). For errant PEI versus baseline, the subsidiary ANOVA (group [half/twice]; encoding duration [30 s/60 s/120 s]; PEI [errant/baseline]) revealed main effects of encoding duration, $F(2,188)=26.23, p<.001, \eta_{\mathrm{p}}{ }^{2}=.218, M S E=$ 244.5, and PEI, $F(1,94)=79.62, \eta_{\mathrm{p}}{ }^{2}=.459, M S E=298.5$, but the encoding duration $\times \mathrm{PEI}$ interaction was not significant, $F<1$, and neither was the three-way interaction between group, encoding duration, and PEI, $F=2.44, p=.09$.

The results from these two subsidiary ANOVAs indicate that group differences in the effect of encoding duration are

Table 1 Percentages of participants ( $n=48$ per group) who ranked their memory as best/worst according to the encoding duration of each image

\begin{tabular}{|c|c|c|c|c|}
\hline Participant Group & Subjective Memory Rating & $30 \mathrm{~s}$ & Encoding Duration $60 \mathrm{~s}$ & $120 \mathrm{~s}$ \\
\hline \multirow[t]{2}{*}{ Half } & Worst & $70.8 \%$ & $10.4 \%$ & $18.7 \%$ \\
\hline & Best & $12.5 \%$ & $33.3 \%$ & $54.2 \%$ \\
\hline \multirow[t]{2}{*}{ Twice } & Worst & $62.5 \%$ & $16.6 \%$ & $20.8 \%$ \\
\hline & Best & $12.5 \%$ & $29.2 \%$ & $58.3 \%$ \\
\hline
\end{tabular}

Note. "30 s," "60 s," and "120 s" refer to the within-subjects manipulation of encoding duration. "Half" versus "twice" refers to the betweensubjects manipulation of beliefs about the relative encoding duration of one's partner 


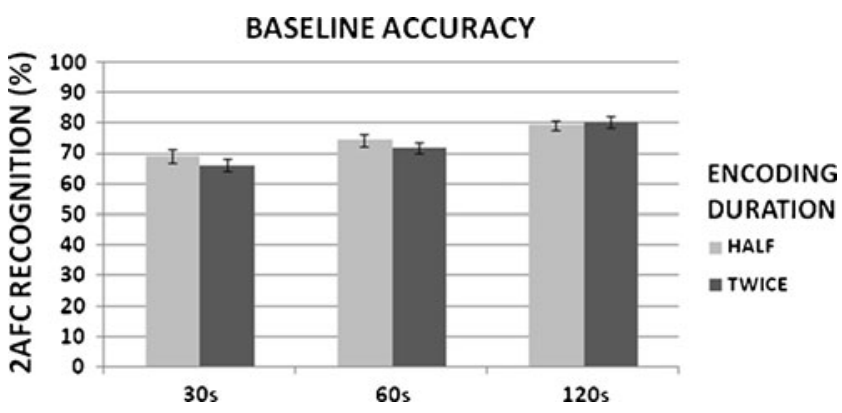

Fig. 1 Two-alternative-forced choice (2AFC) percentages of recognition in the baseline (i.e., no response from partner) condition $( \pm 1$ SEM)

not specific to the accurate or the errant PEI conditions. Hence, the three-way interaction in our original ANOVA reported above does not reflect differential patterns of conformity in each group according to encoding duration within either the accurate or the errant PEI conditions alone. The group differences must therefore be driven by a dissimilarity in the effect that encoding duration has upon the "spread" of performance between the accurate and errant PEI conditions. This interpretation was confirmed by a further subsidiary ANOVA upon the correct 2AFC performance data (group [half/twice]; encoding duration [30 s/60 s/120 s]; PEI [accurate/errant]). The ANOVA gave a significant three-way interaction between group, encoding duration, and PEI, $F(2,188)=6.37, p=.002, \eta_{\mathrm{p}}{ }^{2}=.063$, $M S E=170.4$, which qualified significant main effects of encoding duration, $F(2,188)=12.12, p<.001, \eta_{\mathrm{p}}{ }^{2}=.114$, $M S E=232.1$, and PEI, $F(1,94)=119.52, \eta_{\mathrm{p}}{ }^{2}=.56, M S E=$ 505.6, and a significant encoding duration $\times$ PEI interaction, $F(2,188)=3.61, p=.029, \eta_{\mathrm{p}}{ }^{2}=.037, M S E=170.4$.

We employed a final pair of subsidiary ANOVAs to elucidate the effect of encoding duration upon the spread of performance between the accurate and errant PEI condi-

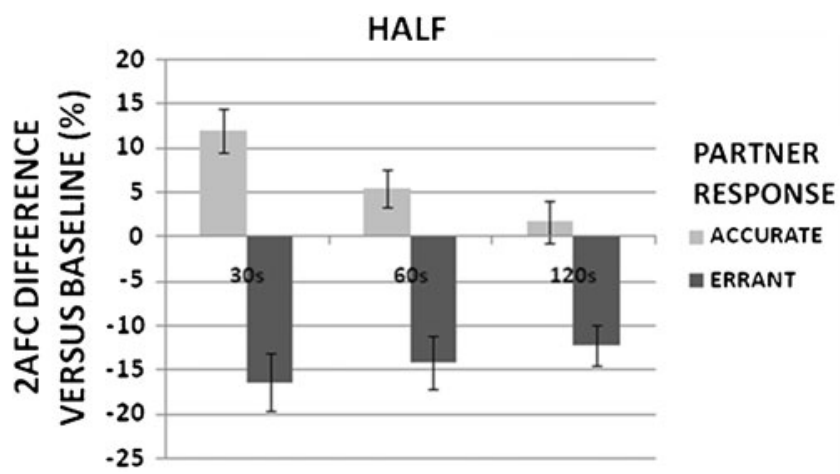

Fig. 2 Correct two-alternative forced choice (2AFC) percentages of performance $( \pm 1 S E M)$ from participants who believed that they had encoded each scene for half as long as their partner. The data show performance following exposure to accurate and errant virtual partner responses, relative to baseline (i.e., accurate minus baseline and errant minus baseline)

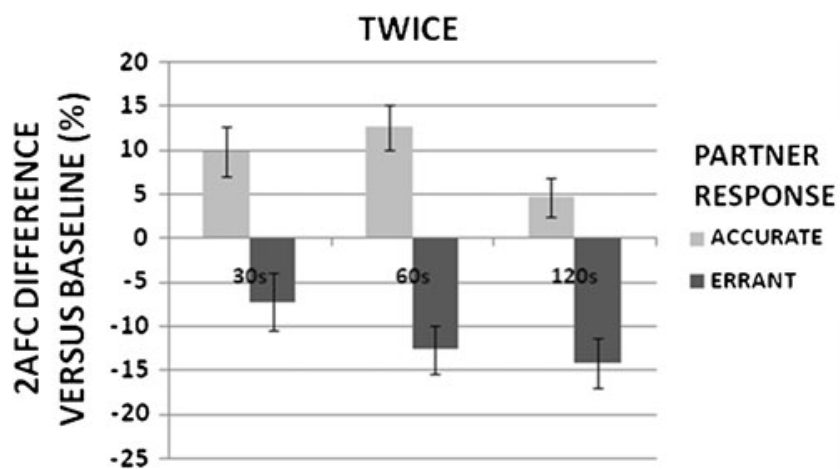

Fig. 3 Correct two-alternative forced choice (2AFC) percentages of performance $( \pm 1 S E M)$, relative to baseline, from participants who believed that they had encoded each scene for twice as long as their partner

tions in each group. In the half group, the ANOVA (encoding duration $\left[\begin{array}{llll}30 & \mathrm{~s} / 60 & \mathrm{~s} / 120 & \mathrm{~s}\end{array}\right]$; PEI [accurate/ errant]) gave a significant main effect of encoding duration, $F(2,94)=5.66, p=.005, \eta_{\mathrm{p}}^{2}=.108, M S E=$ 219.3 , and of PEI, $F(1,47)=58.3, p<.001, \eta_{\mathrm{p}}{ }^{2}=.554$, $M S E=525.3$, and both were qualified by a significant interaction between encoding duration and PEI, $F(2,94)=$ 8.76, $p<.001, \eta_{\mathrm{p}}{ }^{2}=.157, M S E=143.7$. Paired samples $t$ tests then revealed that conformity was significantly enhanced in the $30-\mathrm{s}$ versus 120 -s condition, $t(47)=$ 4.93, $p<.001, S E M=2.9$, and in the 30 -s versus 60 -s condition, $t(47)=2.51, p=.015, S E M=3.5$, but that conformity in the 60- and 120-s conditions did not differ significantly, $t(47)=1.14, p=.16$. In the twice group, the subsidiary ANOVA (encoding duration $[30 \mathrm{~s} / 60 \mathrm{~s} / 120 \mathrm{~s}]$; PEI [accurate / errant]) gave significant main effects of encoding duration, $F(2,94)=6.49, p=.002, \eta_{\mathrm{p}}{ }^{2}=.121$, $\mathrm{MSE}=244.9$, and of PEI, $F(1,47)=61.34, p<.001, \eta_{\mathrm{p}}{ }^{2}=$ $.566, M S E=486.0$, but the interaction between these two factors was not significant, $F=2.24, p=.11$.

In summary, our analyses demonstrate that participants in the half group conformed significantly more often to their partners' memories of the short-duration scene than for both of the other two scenes. The majority of participants in the half group also believed that their memory was worst for the short- (30-s) duration scene, and, more objectively, these participants showed a significant drop in baseline accuracy for the short-duration scene versus both the medium- and longduration scenes. The majority of participants in the twice group believed that their memory was worst for the short-duration scene and also showed significant drops in their baseline performance from the longthrough to the medium- and the short-duration scenes. But, in marked contrast to the half group, this constellation of factors did not produce any detectable increase in conformity within the twice group. 


\section{Discussion}

Our findings reveal that memory conformity can be manipulated by changing a person's perception of how he or she encodes information, relative to his or her dyad partner. Our findings therefore replicate, using virtual confederate methods, the prior work of Gabbert et al. (2007; and see French et al., 2011). We go beyond this prior work, however, by demonstrating that beliefs about another person's memory do not always act to enhance conformity. Instead, a more strategic reliance upon another person's memory was revealed, such that conformity was selectively enhanced in the condition where participants had the briefest (30 s) time to encode the picture stimuli. This increased tendency to conform was specific to participants who believed that they had encoded all scenes for half as long as their partner and was not present in participants who believed that they had encoded all scenes for twice as long as their partner. The specificity of this effect occurred despite a number of similarities between the two groups within the subjective ratings data and within the baseline performance conditions. These additional data show that objective drops in baseline accuracy (see Fig. 1) and subjective reductions in the perceived quality of one's own memory per se (see Table 1) are not sufficient in and of themselves to enhance one's reliance on the memory of another person. If they were sufficient, we should have seen enhanced conformity in the short-duration condition in the twice group. We conclude that beliefs about memory in one's self and in one's partner act in concert, exposing the social metacognition that underlies informational motivations to conform (Wright et al., 2010; Wright \& Schwartz, 2010).

As was described in the introduction, Gabbert et al. (2007) showed previously that memory conformity can change as a function of beliefs about the accuracy of one's partner. Participants who believed that they had encoded for half as long as their partner conformed more than participants who believed that they had encoded for twice as long as their partner, despite the fact that overall accuracy in both participant groups was statistically identical. In the present experiment, we show that even when consistent changes in baseline accuracy do occur (see Fig. 1), this does not lead to identical changes in the pattern of conformity within each group. Processes modulating conformity therefore do not respond in an obligatory way to changes in one's own memory accuracy if other relevant information about one's partner is available-for example, information about their relative encoding duration (cf. Baron et al., 1996; Roediger et al., 2001; Walther et al., 2002). Hence, strategic processes that underlie shifts in conformity may function independently of processes that determine memory accuracy per se.
Other studies have also reported findings that support functional independence between processes underlying conformity and processes affecting memory accuracy per se. For example, we have previously observed equivalent levels of conformity in groups of younger and older adults whose memory accuracy showed significant age-related impairments (Gabbert, Memon, \& Allan, 2003). Wright, Gabbert, Memon, and London (2008) have also demonstrated a link between strategic shifts in performance and resulting levels of conformity, using instruction manipulations to alter response criterion. These results lend support to the conclusion that processes underlying conformity are highly strategic in nature and can function independently of processes affecting the accuracy of one's memory. But it is worth pointing out one caveat from our own findings. In the twice group, some evidence of sensitivity to encoding duration did come from our analysis of performance following exposure to accurate PEI. Here, twice group participants showed a reduced tendency to conform in the longest encoding duration condition, as did participants in the half group (see Figs. 2 and 3), and this was associated with a significant encoding duration $\times$ PEI interaction that did not involve the group factor (see the Results section). This finding suggests at least some common sensitivity in both groups to the changes in memory accuracy produced by the manipulation of encoding duration.

We conclude that one's susceptibility to social influence may be strategically moderated by the need to maintain a stable and reasonably accurate memory, which seems to be a highly reasonable way for us to function as social beings capable of supplementing our own knowledge with that of other people. The present findings are therefore somewhat analogous to earlier work by Allen and Levine (1971), who showed that conformity to a group can be significantly reduced if one has a partner within the group who offers a "valid" form of social support against the group consensus. The participant's partner was actually an experimental confederate whose "validity" was reduced in one condition by wearing eyeglasses that gave the impression of severely limited vision. The participants' task involved a variety of Asch-type (e.g., Asch, 1956) perceptual and semantic judgments about line length, factual judgments, and opinion statements to be given after three other individuals - one of whom was the designated partner-had given their unanimous response. Allen and Levine found that conformity to the group answer was significantly reduced when the validity of a partner had not been undermined. They concluded that the partner may provide an independent assessment of reality that offers social-that is, interpersonal- support against conformity to a group consensus.

Our present findings extend Allen and Levine's (1971) prior work, by suggesting that the "validity" or perceived expertise of another person may be specific to certain past contexts that define the scope of their relative expertise and 
in which their social influence over our decision making is heightened (see French et al., 2011, for a similar conclusion). The presence of the baseline differences in accuracy for each image and the manipulation check data suggest that our participants were well aware that their own memory was better for the long- versus short-duration images. But in the twice group, this knowledge alone was not sufficient to provoke increased reliance upon one's partner to supplement one's own memory judgments. Thus, previously separate but complementary lines of work, dealing with changes in one's own accuracy and with perceptions of accuracy in other people, have been brought together to provide strong evidence that memory conformity arises from a trade-off that integrates beliefs about memory in one's self and other people.

More specifically, it appears that beliefs about the effect of encoding durations upon memory in one's self and other people act in concert. This leads us to a final point for future work on conformity. The present results, in our view, strongly suggest a form of social metacognition whereby knowledge of the conditions that modulate our own memory is systematically applied to the functioning of memory in other people. This may reflect a general tendency to use one's own mental states to simulate those of other people. We suggest that this simulationist approach to social cognition (see, e.g., Nickerson, 1999; Tamir \& Mitchell, 2010) not only may provide a general explanatory framework relevant to informational social influences, but also may lead to further, novel avenues for research upon normative social influence. Models that seek to explain and combine normative and informational social influences (e.g., Wright et al., 2010; Wright \& Schwartz, 2010) may, therefore, have much to gain from future work exploring what the simulationist perspective has to offer.

\section{References}

Allan, K., \& Gabbert, F. (2008). I still think it was a banana: Persistent lies and forgettable truths. Acta Psychologica, 127, 299-308

Allen, V. L., \& Levine, J. M. (1971) Social support and conformity: The role of independent assessment of reality. Journal of Experimental Social Psychology, 7, 48-58.

Asch, S. E. (1956). Studies of independence and conformity: A minority of one against a unanimous majority. Psychological Monographs, 70, 416.

Baron, R. S., Vandello, J. A., \& Brunsman, B. (1996). The forgotten variable in conformity research: Impact of task importance on social influence. Journal of Personality and Social Psychology, 71, 915-927.

Bodner, G. E., Musch, E., \& Azad, T. (2009). Re-evaluating the potency of the memory conformity effect. Memory \& Cognition, 37, 1069-1076.
Cialdini, R. B., \& Goldstein, N. J. (2004). Social influence: Compliance and conformity. Annual Review of Psychology, 55, 591-621.

Deutsch, M., \& Gerard, H. G. (1955). A study of normative and informational social influence upon individual judgement. Journal of Abnormal and Social Psychology, 59, 204-209.

French, L., Garry, M., \& Mori, K. (2008). You say tomato? Collaborative remembering leads to more false memories for intimate couples than for strangers. Memory, 16, 262-273.

French, L., Garry, M., \& Mori, K. (2011). Relative - not absolutejudgements of credibility affect susceptibility to misinformation conveyed during discussion. Acta Psychologica, 136, 119-128.

Gabbert, F., Memon, A., \& Allan, K. (2003). Memory conformity: Can eyewitnesses influence each other's memories for an event? Applied Cognitive Psychology, 17, 533-543.

Gabbert, F., Memon, A., Allan, K., \& Wright, D. B. (2004). Say it to my face: Examining the effects of socially encountered misinformation. Legal and Criminological Psychology, 9, 215-227.

Gabbert, F., Memon, A., \& Wright, D. B. (2007). I saw it for longer than you: The relationship between perceived encoding duration and memory conformity. Acta Psychologica, 124, 319-331.

Hope, L., Ost, J., Gabbert, F., Healey, S., \& Lenton, E. (2008). "With a little help from my friends...".: The role of co-witness relationship in susceptibility to misinformation. Acta Psychologica, 127, 476-484.

Macrae, C. N., \& Bodenhausen, G. V. (2000). Social cognition: Categorical person perception. British Journal of Psychology, 92, 239-255.

Meade, M. L., \& Roediger, H. L., III. (2002). Explorations in the social contagion of memory. Memory \& Cognition, 30, 995-1009.

Nickerson, R. S. (1999). How we know-and sometimes misjudgewhat others know: Imputing one's own knowledge to others. Psychological Bulletin, 125, 737-759.

Reisberg, D., Pearson, D. G., \& Kosslyn, S. M. (2003). Intuitions and introspections about imagery: The role of imagery experience in shaping an investigator's theoretical views. Applied Cognitive Psychology, 17, 147-160.

Roediger, H. L., III, Meade, M. L., \& Bergman, E. T. (2001). Social contagion of memory. Psychonomic Bulletin \& Review, 8, 365371.

Schneider, D. M., \& Watkins, M. J. (1996). Response conformity in recognition testing. Psychonomic Bulletin \& Review, 3, 481-485.

Skagerberg, E. M., \& Wright, D. B. (2009). Sibling differentials in power and memory conformity. Scandanavian Journal of Psychology, 50, 101-107.

Tamir, D. I., \& Mitchell, J. P. (2010). Neural correlates of anchoringand-adjustment during mentalising. Proceedings of the National Academy of Sciences, 107, 10827-10832.

Tousignant, J. P., Hall, D., \& Loftus, E. F. (1986). Discrepancy detection and vulnerability to misleading postevent information. Memory \& Cognition, 14, 329-338.

Walther, E., Bless, H., Strack, F., Rackstraw, P., Wagner, D., \& Werth, L. (2002). Conformity effects in memory as a function of group size, dissenters and uncertainty. Applied Cognitive Psychology, 16, 793-810.

Wright, D. B., Gabbert, F., Memon, A., \& London, K. (2008). Changing the criterion for memory conformity in free recall and recognition. Memory, 16, 137-148.

Wright, D. B., London, K., \& Waechter, M. (2010). Social anxiety moderates memory conformity in adolescents. Applied Cognitive Psychology, 24, 1034-1045.

Wright, D. B., \& Schwartz, S. L. (2010). Conformity effects in memory for actions. Memory \& Cognition, 38, 1077-1086. 